\title{
EL PENSAMIENTO: UNA DEFINICIÓN INTERCONDUCTUAL
}

\author{
Alberto Melgar Segovia
}

\begin{abstract}
RESUMEN
El pensamiento ha sido definido como si ocurriera en dimensiones distintas a la objetiva. El pensar estaría conformado por procesos internos no susceptibles de observación. Esta traba metodológica fue superada en las teorías de Skinner y Vigotsky. Skinner propuso la introducción de estímulos objetivos en la cadena de respuestas en el pensar. Vigotsky enfatizó la paulatina intervención de instrumentos en la estructuración de la conducta del pensar, principalmente el lenguaje. No obstante estas definiciones caracterizan al pensar como una 'autogeneración de estímulos', con muchas similitudes a los estímulos «internos» de las definiciones mentalistas. Kantor define al pensamiento como la manipulación de conductas implícitas y manifiestas, pero el concepto de 'manipulación' hace recordar enfoques mentalistas. Ribes señala que el pensar es un tipo especial de relación de la cual participa la conducta. Se describe el pensar como «autosustitución referencial». "Sustituir" equivale a transformar que significa tener la capacidad para desligar conductas particulares de su correspondencia funcional con las contingencias fisicas presentes y, conforme a esto, ser capaz de ligar estas conductas a circunstancias no presentes en la situación actual. Sin embargo, este tipo de interacción no necesitaría niveles sustitutivos referenciales pues la 'sustitución' entendida como 'transformación' de contingencias ya esta presente en interacciones más simples. Proponemos una definición alternativa del pensar, como el desarrollo de nuevos «sentidos» ante las situaciones. Además, se propone una metodología para el estudio del pensamiento. Esta es una variación del método de igualación a la muestra, presentando las conductas que conforman el pensar como alternativas a escoger para resolver las tareas. El método llamado «Igualar» posibilito mejores desempeños que el de igualación, además de ser mas eficaz para facilitar avances cuando se introducían items de transición. Sin embargo las diferencias no fueron significativas estadísticamente.
\end{abstract}

\begin{abstract}
The thinking has been defined as it happens in a different dimension from the objective dimension. The thinking would be formal for internal processes unable to be observed. This methodological block was over for the theories of Skinner and Vigotsky. The first one proposed the introduction of objective stimuli in the responses chains of the Thinking process. Vigotsky remarked the slow intervention of tools in the structuration of the thinking behavior, meanly the language. However, these definitions describe the Thinking as an 'stimuli autogeneration', with many similarities to the internal stimuli of the cognitive definitions. Kantor defined the thinking as the manipulation of implicit and over ( responses, but the concept of 'manipulation' reminds mentalistic points of view. Ribes points that the thinking is a special type of relation in which participates the behavior. 'He describes the thinking as 'referencial autosustitution'. 'The substitution' equals to transform, that means the ability to dettache particular behaviors from their functional correspondence to physical contingencies in the real situation. However, this type of interaction would not need substitutive referencial levels because the substitution, understood as 'transformation' of contingencies, is already present in single situations. We propuse an alternative definition of thinking as the development of new meanings before situations. Besides, we propose a methodology for the study of the thinking process. This is a variation of the matching to sample method, that introduces the behaviors conforming the thinking as alternatives to choose for solving the tasks. This method called 'Igualar' permited better achievements than the 'matching lo sample' method, besides of being more effective to facilitate improvements when transition items were set. However, the differences were not meaningful from the statistics.
\end{abstract}


El pensamiento ha sido descrito en la psicología como la capacidad de planear y dirigir en forma oculta una conducta posterior, lo que prevenía de errores o permitía postergar las acciones para posibilitar adaptaciones mejores en duración y efectividad. Este rasgo de no apariencia hizo que en ulteriores análisis del significado de "pensar" se ponga un énfasis decisivo en la inobservabilidad del pensamiento.

En efecto, en diferentes teorías psicológicas se le ha descrito como un proceso que ocurre en la instancia interna de la mente. Si se le define como cambios en los estados de información (González, 1991 p. 124), estos cambios que sufre la información son a merced de varios mecanismos mentales ocultos. Si bien el pensamiento tiene un carácter no aparente es justamente este rasgo el que lo ha confinado, para estas teorías, a un mundo interno. $\mathrm{Y}$ esto es así, porque proponer su lugar en un mundo alejado del alcance del ojo humano, deja abierta la posibilidad de describirlo en términos muy especulativos. El intento de dar a esta instancia inobservable un carácter material -señalando que se trata de un mecanismo neural o cibernético- no ha resuelto el problema porque se sigue concibiendo su ocurrencia en instancias distintas a la interacción real entre el sujeto y los objetos.

El carácter no observable del pensamiento no es razón para confinarlo a un mundo no material, porque este rasgo indica simplemente otra forma de relacionarse al ambiente. Es un modo de relacionarnos con objetos y situaciones no presentes en el momento actual y, por tanto, sin producir ningún efecto en los objetos (Kantor, 1924-26)

No se puede juzgar al pensamiento con criterios de observabilidad -diciendo por ejemplo que por no ser visible pertenece a un mundo interno- porque la observabilidad no es una de las propiedades del pensamiento. Siendo así, es parte de la experiencia personal de la que solo puede dar cuenta el sujeto. Pero incluso como experiencia personal, son hechos que no podemos ver a través de ningún órgano sensorial porque no son sensibles sensorial mente. Gran parte de las conductas que conforman el pensamiento son las conductas implícitas, formas derivadas de la percepción. Las percepciones, son reacciones sensoriales no observables pero sí evidentes pues el objeto percibido está presente. Cuando el objeto ya no está presente, se les describe como conductas implícitas. Cuando percibimos establecemos en realidad varias relaciones con el ambiente. Respondemos a más estímulos de los que creemos. En el futuro, aun si faltase un componente de la situación original, la conducta similar ocurrirá pues la conducta en cuestión ya no depende de un objeto determinado, sino de otros que han estado cobrando importancia. Entonces, el sujeto parece responder a un estimulo no presente.

Las conductas implícitas se pueden considerar como formas especiales de relaciones. Y sabemos que con frecuencia las relaciones no se observan. Estas relaciones se establecen, a veces de maneras arbitrarias y/o con cierta concordancia con la realidad. Uno de los modos de comprobar su ocurrencia es la inferencia a partir de la observación de sus componentes (si son susceptibles de observar) o el testimonio de la experiencia personal del sujeto que interactúa. (¿Que imaginaste?, ¿Cómo recordaste?, etc.).

Dentro de la misma orientación cognitivista se objeta la caracterización que hace del pensamiento, la teoría del procesamiento de información. El procesamiento de información implica el tratamiento de las diferentes entradas de información que provienen de diferentes 
canales sensoriales (visual, auditiva, etc.). Pero ellas deben asumir la forma de proposiciones para que puedan ser procesadas de manera equivalente. Pero ello implica que hay ciertas reglas internas que puedan darle esa forma. Pero las reglas se explican a partir de otras, en una cadena ad-finitum. Encontrar los algoritmos capaces de traducir las entradas sensoriales a proposiciones es el problema mayor. Pero estos algoritmos, deben apoyarse de todas maneras en términos de algún otro lenguaje que uno es capaz de emplear previamente. ¿Cuál es ese lenguaje? Si se dice que es el que socialmente aprendemos, el ser humano tendría diferentes conjuntos de reglas porque hay diferentes lenguajes, lo cual estaría en contradicción con la idea de que estas reglas mentales son universales. Si se quiere mantener la universalidad de las reglas, se tendría que suponer que estas reglas las tenemos de nacimiento.

Otras Críticas están referidas al cuestionamiento de que términos como «deducción», «inferencia», etc., describen procesos del sujeto que actúa. Estos términos son utilizados con frecuencia en las teorías cognitivistas. Tal noción errónea aparece cuando se piensa que el uso de una expresión, implica que su significado debe acaecer de alguna manera. Estos términos, con frecuencia, sólo son adecuados para la presentación de ideas que ya se poseía y no describen procesos. Cuando demostramos un razonamiento empleamos la frase como «dado tal y cual», «deduje o inferí tal cosa». Al describir como se resolvió algo se emplean términos como «deducir» $o$ «inferir» que son en realidad términos que solo describen lo ya sucedido, no lo que esta sucediendo o lo que va suceder. El mismo error se comete cuando el manejo de términos abstractos se explica por la existencia de una entidad interna llamada concepto que se consulta de vez en cuando para poder actuar. Pero en realidad decimos que tenemos una idea abstracta solo cuando nos comportamos de maneras dadas ante una clase de objeto. Tener una idea del relieve, por ejemplo, es solo poder hacer cosas como leerlos en un mapa, dibujar un mapa que indique el relieve, etc.

Dentro del enfoque cognitivo, la definición de Piaget hace un énfasis más decidido en las relaciones entre sujeto y medio. El pensamiento es un nivel superior de la acomodación y asimilación y que opera con los productos de ellas, los esquemas, que son el resultado de los encuentros asimilativos y acomodativos con el medio. Los esquemas son representaciones interiorizadas de una clase de acciones o desempeños similares. Permiten que una persona haga algo "en su mente" sin comprometerse en una acción manifiesta. Según Piaget, al interactuar con un objeto nuevo, se activa un esquema que permite asimilar tal objeto; a su vez, este contacto hace que se modifique el esquema para que pueda incluir la nueva forma de actuar con el nuevo objeto. Antes de la formación de esquemas, señala Piaget, el sujeto es egocentrista, es decir, no diferencia su mundo personal del mundo externo. Pero en el contacto con el medio encuentra obstáculos que producen cambios de perspectiva, dando origen a asimetrías en la relación entre el sujeto y el entorno. Esta asimetría conducirá en algún punto a considerar al objeto como independiente. El sujeto, luego podrá variar su posición en la relación, establecer relaciones especificas entre él y los objetos o entre los objetos. Este proceso es denominado «descentramiento». «Centrarse» es atender solo un punto perceptivo a la vez, y «descentrarse» es atender mas de un punto a la vez. El sujeto, al atender más de un punto, se da cuenta que los elementos percibidos varían y que su actuar no es independiente sino siempre es sobre «algo». Cuando se atiende a mas de un punto, se puede establecer una continuidad entre los puntos que se atienden, especialmente si constituyen estados. El esquema tiene como objetivo representar esta continuidad de forma mental. El sujeto como ha atendido la transición de los estados y ha establecido una continuidad, puede mentalmente representar la transición al otro estado. El 
sujeto se vale de indicios para establecer esta transición, este proceso; por ejemplo, al alargar una bolita de plastilina, el indicio «reducción del grosor» ayuda a comprender el proceso de alargamiento de la bolita. El mismo esquema se sirve de este indicio para representar mentalmente un regreso al estado original -la bola de plastilina- e inferir la constancia de volumen. Estos indicios lo dan los mismos objetos por lo que se puede decir que es en la interacción con los objetos donde se desarrollan las adaptaciones. El indicio participa en la actuación del esquema, afectando y matizando su desenvolvimiento. En el pensamiento, son los indicios los que permiten que los objetos sean considerados en varios planos posibilitando el desarrollo de conductas que se mueven en varios planos, es decir, permitiendo variadas orientaciones.

Las Teorías Conductistas: En estas teorías, el pensamiento seria una forma no observable de lenguaje, un «habla interiorizada» $\mathrm{o}$ «conducta gobernada por reglas».

Para Vigotsky, por ejemplo, el pensamiento surge a través de algún conflicto del sujeto que obliga para su resolución revelar de la situación aquello que es nuevo. Se suceden entonces actos de significación que toman la forma de palabras, forman un habla. Los actos de significación al asumir la forma de palabras, enriquecen estas palabras formándose numerosos «sentidos», para cada palabra, es decir, dándole una generalidad a cada palabra. Al volver a intervenir estas palabras en el pensamiento, aportan sus variados significados. La emisión de palabras puede pasar de ser externa a interna. En esta descripción del pensamiento, sin embargo, se pone en primer plano el desarrollo de los significados de las palabras, cuando un análisis centrado en las conductas podría explicar mejor dichos eventos: el desarrollo de los significados - las generalizaciones- se dan en situaciones conductuales, los significados de las palabras se observan en condiciones de ocurrencia de comportamientos.

En lo que respecta a Skinner, el pensamiento significa comportarse débilmente tanto que otras personas no lo pueden detectar y la debilidad puede deberse a un control de estímulo deficiente. Así, esta conducta no esta bajo control de las contingencias reales, sino por reglas verbales que especifican contingencias, reproduciendo las propiedades de ciertas condiciones de estimulo. El sujeto actúa como si estuviera en tales contingencias.

Como se ve, tanto en Skinner como en Vigotsky, para definir el pensamiento se apela a un reemplazo de contingencias a través de la autogeneración de estímulos: un lenguaje interiorizado o reglas. Sin embargo, no existen estímulos nuevos ni autogenerados ni internalizados por el sujeto. Muchas de estas ideas referidas a la autogeneración vienen del hecho de la intervención de estímulos lingüísticos como mediadores en la cadena de respuestas que constituirían el recordar o solución de problemas. Pero, si consideramos no a los mediadores sino a la generación de mediadores, es decir, el responder verbalmente, como el enlace en dichas cadenas veremos que, en realidad, todos estos eventos son sucesiones de respuestas. Se dice que las palabras «auto generadas» presentan verbalmente una situación pasada para que el sujeto actúe «como sí» estuviera en esa situación. Pero creemos que la situación evocada no esta presente como estímulos, solo están las conductas que se asemejan a prácticas lingüísticas ya hechas en situaciones anteriores, trayendo significaciones que se encuentran pertinentes en la actualidad. Si antes de entrar a una habitación, me digo "toca la puerta", lo que ocasiona mi respuesta posterior no es el estímulo verbal «toca la puerta» sino la conducta verbal. No es como cuando al acercarnos a fuego; éste, como estimulo, nos hace retirar la mano. Es una conducta verbal la que facilita la ocurrencia de otra, por ejemplo un recuerdo, una solución. 


\section{El pensamiento desde el enfoque Interconductual}

Las definiciones del pensamiento del enfoque interconductual se insertan en una concepción especial sobre eventos psicológicos. Los eventos psicológicos no son simples conductas como movimiento sino que pueden incluir con juntos de conductas. Una caracterización mejor definiría a los eventos psicológicos como interacción, donde las respuestas y estimulas se afectan unos a otros. Estas interacciones son un tipo de relación. Las relaciones se han conceptualizado como la concordancia entre dos sucesos: cuando un suceso ocurre, concomitantemente en el tiempo y/o en el espacio, también otro ocurre. El sujeto se mueve y forma parte de estos conjuntos de relaciones físicas, químicas, biológicas, psicológicas, sociales. Las relaciones psicológicas incluyen a todas las formas de adaptación singulares, idiosincrásicas desarrolladas en el transcurso de la vida de un sujeto. Lo biológico constituye solo el soporte con que nacemos, especialmente ciertas «disposiciones» a partir de los cuales desarrollamos formas de interrelación con el medio. Las «disposiciones» permiten remarcar el concepto de interacción, pues solo algunas disposiciones serán pertinentes en la interacción de acuerdo a si ciertos elementos en la situación aparecen. Para representar estas relaciones en varios niveles, se ha propuesto el modelo de campo. Este representa teóricamente la interacción de múltiples factores; en él se observa que la conducta es continua, causante de y causada por varios factores, y donde el organismo actúa como un todo, físico, químico, biológico y social.

\section{Formación del pensamiento}

Lo psicológico evoluciona principalmente en sus características implícitas. Es decir, va de las respuestas a estímulos presentes a la formación de respuestas cuyos objetosestímulo están ausentes. En este tránsito, las respuestas manifiestas pierden su papel principal en la interacción, papel que es asumido por otras relaciones, más bien inaparentes, que se han ido formando en la interacción manifiesta. Al darse las conductas inaparentes lógicamente los efectos sobre los objetos desaparecen. Esta génesis se inicia en el periodo en que el sujeto reacciona a ciertos rasgos del objeto como un todo, luego ante muy pocos, posteriormente ante rasgos no pertenecientes al objeto y finalmente a estímulos convencionalmente asignados a dicho objeto, siendo los verbales los más comunes. Al no haber efectos sobre los objetos, el único cambio que se produce es en la postura del sujeto ante estos objetos. Las respuestas implícitas sirven como mecanismos para desligar las reacciones de significación de ciertas situaciones, posibilitando la manipulación e integración de estos significados desligados para usarlos como reacciones anticipatorias a determinados ajustes. Señala Kantor, que es la posibilidad de que un objeto sea substituido lo que hace probable una extensa serie de posibles conexiones con los objetos. 


\section{Definiciones}

Para Kantor (1924-26), el pensamiento consiste en la manipulación manifiesta e implícita de cosas y situaciones como procesos preliminares frecuentemente dirigidos a prácticamente otras actividades inmediatas ... instrumentales que hacen el camino o proveen los detalles para una actividad o ajuste que seguirá en un momento apropiado.

Otra definición interconductual más moderna del pensamiento es la de Ribes (1990). Coincide con Kantor en que el pensar, como concepto, no se refiere a una clase especial de conducta, sino más bien a un tipo especial de relación de la cual participa la conducta. El pensar tiene su origen en la conducta sustitutiva: conducta convencional que transforma las situaciones contingenciales a las cuales uno o más individuos en relación están respondiendo. En la «sustitución», un referidor habla sobre un referente a un referido, y al hacer ello transforma las contingencias referidas, le da un matiz propio a su descripción del referente. Esta posibilidad de hacer que un sujeto interactúe con un referente de forma indirecta, permite que el otro pueda interactuar con referentes no presentes y que cuente con información que de otra manera no estaría disponible. Así, el responder se puede ligar a una situación no presente. Esto es posible sólo a través de respuestas lingüísticas. La transformación implica que el sujeto actúa «como si» estuviera en otra situación; o también en términos de lo que le dicen de la situación. En este último caso, se vuelve dependiente de conducta referencial e independiente de cualquier situación específica.

Según Ribes la sustitución se vuelve pensar. ¿Cuándo?, cuando una respuesta convencional produce las condiciones necesarias para relacionar eventos de estimulo convencionales independientes. Dos eventos de estímulos convencionales contingentes a sus respectivas respuestas convencionales, se vuelven interdependientes por una tercera respuesta convencional que media entre ellas. Y esto sucede cuando el mismo sujeto puede hacer esta mediación. El pensar es, pues, conducta «autosustitutiva». En el pensar el sujeto transforma las mismas contingencias para él y a las que solo él responde.

Una última definición es la de Roca (1993). Para Roca, la naturaleza se presenta como «acciones», comportamientos, actos. Es decir, la naturaleza, el ser, son actos. Estos actos se dan con una regularidad, por ello se les llama consistencias. Lo psicológico es una forma de «ser», son comportamientos que se dan también como consistencias. Lo psicológico es un conjunto de «constancias» que se dan dentro de otras constancias llamadas «universos camportamentales» que pueden ser biológicos, psicológicos o sociales. Su base son los comportamientos biológicos. Un tipo de comportamiento es el Pensar, que es un conjunto de constancias ante las constancias estimulativas socio-culturales o problemas convencionales.

\section{Criticas a las definiciones Interconductuales del Pensamiento}

Kantor enfatizó en el rasgo inclusivo de varias conductas a fin de adaptarse a una situación compleja. Entre esas conductas ubicó como preponderantes a las conductas implícitas. La primera observación es la confusa recurrencia a la «manipulación» parecida a la realizada por las teorías mentalistas. Además, la simple combinación de conductas no aclara como es que el sujeto llega a combinar conductas, como es que forma tan compleja de interacción aparece y que principio rige la sucesión particular de conductas en el pensar. Asimismo, no se ve como conductas tan diferentes se combinan. Las conductas implícitas y manifiestas pertenecen a niveles diferentes, lo que no solo se refiere a su observabilidad 
sino a la diferencia del material con que trabaja (estímulos de ajuste y sustitutivo), a la cantidad de referencias del objeto y el nivel de desligamiento con respecto a tales objetos.

\section{La teorización de Ribes también muestra deficiencias:}

\section{La "Transformación" de Contingencias para uno mismo:}

Se parte de la idea de que la «autosustitución de contingencias» se origina en la «sustitución de contingencias» para otros. Esta última, supone una transformación de las contingencias actuales en base a otras no presentes. El sujeto reacciona a los objetos también en términos de las características no presentes del objeto. Es decir, hay «transformación de contingencias para uno mismo» en la «sustitución de contingencias» para otros (comunicación): por ejemplo, cuando el sujeto pide algo a alguien utiliza sus respuestas verbales ante un referido como «herramientas» para conseguir algún objeto presente en la situación. En esa situación transforma la situación para el referido, pone en contacto al referido con una situación a la que tal vez no esta atendiendo. Pero para ello transforma sus propias contingencias para incluir sus palabras como «herramientas de comunicación». Cuando el hablante le pide un objeto al oyente, se dan en realidad dos tipos de interacciones: una orden como acto referencial que hace el referidor y una respuesta de entendimiento que hace el referido. Pero el entendimiento, también se da en el referidor: «que digo» y «para que». Este entendimiento implica que el referidor asigna un rol al referido como mediado, además de definir lo que se quiere hacer. El referidor asignando un rol al referido, a sus palabras como herramientas, y determinando lo que quiere, está transformando su propia contingencia. Cuando solo se quiere contar y no inducir, el objetivo instrumental es justamente referenciar, 10 que está ligado a necesidades sociales. Hay que notar que el lenguaje aparece en circunstancias instrumentales donde la transformación de contingencias es real, con los objetos referidos presentes, por ejemplo, cuando se pide un objeto.

Ahora, este uso instrumental del lenguaje se asemeja a una situación más simple: la utilización de un instrumento material para alcanzar un objeto. Ya que el sujeto solo guarda contacto perceptivo con el objeto, transforma su relación con dicho objeto cuando utiliza una herramienta para alcanzarlo. Este es un evento claro de pensamiento pero que se dan con los estímulos de ajuste presentes. El lenguaje aparece en circunstancias similares pero posteriormente por lo que no se puede postular la existencia del pensamiento a partir del lenguaje. Finalmente, la transformación en terminas de las propiedades lingüísticas se incluye en el pensamiento pero no es el «pensamiento».

\section{Transformación en términos de situaciones pasadas:}

Ribes señala que una de las condiciones en que la conducta sustitutiva es autosustitutiva es cuando la conducta del individuo afecta su propia conducta en las condiciones presentes en términos de situaciones previas. Sin embargo, esta característica no es privativa de la autosustitución. Lo mismo podemos decir de situaciones en la que se operan transformaciones efectivas, como la utilización de herramientas materiales. Este uso tuvo su origen en circunstancias pasadas, pero ocurre varias veces luego porque tienen un 
sentido para el sujeto. Su ejecución posterior sugiere por lo menos que el sujeto le ha dado al objeto del evento anterior un sentido instrumental, que luego operará en otra situación. Cuando aprendió el uso de la herramienta, respondió a la relación con textual de herramienta y objeto o su respuesta los relacionó, pero ello no asegura que ante una situación posterior una relación similar entre herramienta y objeto ocurrirá; sin embargo, esa conducta instrumental ocurre de nuevo. Para explicar esto, se ha señalado que la relación se fortalece en varios ensayos o hay asociación fuerte la primera oportunidad. Tal fortalecimiento no se puede dar de manera natural: a) Porque tiene poca oportunidad de suceder, b) Debería presentarse las situaciones para su fortalecimiento de manera inmediata y poco espaciada, c) La asociación se da a pesar de ser los estímulos muy distintos (una vez es un palo y otra un juguete).

Una mejor explicación supone un entendimiento por parte del sujeto del objeto mediador como instrumento para alcanzar algo luego de los primeros intentos. Explica su ocurrencia a pesar de su poca frecuencia, el lapso pues las situaciones se pueden generalizar como relacionadas a instrumentos y la generalización del objeto instrumental. En resumen, la actuación en términos de situaciones pasadas no se da solo en la autosustitución.

\section{EI sujeto como referido y referidor:}

Otra objeción a la «autosustitución» se refiere a su definición como contingencia donde el sujeto reacciona como referido y referidor. En este caso, el sujeto se habla acerca de sus propias conductas referenciales ante eventos. Es una transformación no de las contingencias directas del sujeto sino de las referencias del sujeto a los objetos, siendo netamente lingüística. Pero esto también caracteriza al recordar donde si bien se requieren conductas de pensamiento, el fin no es dar un nuevo sentido sino el sentido que se ha olvidado.

\section{Pensar en grupo:}

Al considerar al pensar como autorreferencia se asume que el evento solo ocurre en un individuo. Sin embargo, cuando «pensamos en grupo» varios sujetos median sus respuestas referenciales a través de alguna respuesta referencial que uno u otro dan cumpliéndose el rasgo de la mediación de respuestas convencionales implicado en la autosustitución. Pero ya no es «autosustitución» porque no es solo para uno mismo.

\section{Memoria como pensamiento:}

Ribes incluye como un tipo de autosustitución el caso donde el sujeto reacciona más a las situaciones anteriores que a las actuales, en este caso el sujeto <<recuerda〉>, está $<<$ como si $>>$ estuviera en otra situación. Pero así, el recordar sería una forma de pensamiento y sabemos que los recuerdos no generan ningún nuevo sentido.

\section{Coordinación de respuestas}


Ribes señala que la «autosustitucion» solo es posible en niveles lingüísticos. Sin embargo, en el pensamiento interviene conducta manifiesta e implícita, no solo las implícitas.

Roca (1993) intenta una definición del pensar, enfatizando y llevando hasta sus últimas consecuencias la naturaleza interactiva de lo psicológico, dejando en un plano secundario sus características morfológicas. Lo principal es que el pensar es la ubicación en un universo comportamental social. Este universo permite que un mismo conjunto de estímulos se presente en distintas dimensiones significativas, por lo que cada pensamiento implicaría una ubicación distintiva en una dimensión. Sin embargo, no se puede describir estos universos solo como campos convencionales, desligándolo de su origen en circunstancias concretas.

\section{Reformulación de las definiciones interconductuales de pensamiento}

\section{Definición de evento psicológico}

La caracterización hecha por Kantor de los eventos psicológicos así como la de otros teóricos dentro de la Psicología Interconductual nos permite, en primer lugar, ver a dichos eventos como el conjunto de interacciones de distintos factores que conforman un campo. $\mathrm{Y}$ en segundo lugar, ver que cada evento es una unidad especifica. Dicha unidad la da el «significado psicológico» de cada evento, concepto con el cual queremos tomar en cuenta la dimensión comportamental del evento y el propósito adaptativo que tiene. Dado que se delimita cada evento solo en base a estos criterios, puede ser de poca o larga duración, incluir solo un segmento conductual o varios. Creemos que es el significado psicológico lo que determina la naturaleza morfológica y funcional de los segmentos que conforman un evento. El objeto de estudio de la psicología debería ser un episodio psicológico con una determinada función relacionada a ciertos fines y con ciertas características morfológicas. El pensar debe ser sometido a este tipo de descripción.

\section{La evolución de las conductas:}

Se ve el origen de las conductas implícitas en las conductas manifiestas. Las conductas manifiestas incluyen múltiples rasgos que conforman la función principal de conductas implícitas. El supuesto que sigue es que dichos rasgos se independizaron conformando conductas implícitas. Una conducta manifiesta ocurre en ausencia de un estimulo y con el tiempo la misma respuesta cambia morfológicamente teniendo solo parte de la apariencia anterior. Más adelante, las respuestas se reorganizan en otros sistemas reactivos (como los verbales), desligándose así, de los eventos originales tanto del lado de los estímulos como de las respuestas. La posibilidad de responder ante objetos no presentes se explica considerando a las conductas implícitas como prolongaciones de respuestas cuando el estímulo ha desaparecido, ocurre ahora ante estímulos sustitutivos. Los estímulos sustitutivos evolucionan hasta tomar la forma de símbolos. 


\section{La característica principal del pensamiento}

La característica principal que encontramos en el pensamiento es que está encaminado a producir nuevos sentidos, ya sea para conductas posteriores o como conducta final. Es por ello que implicaría «transformar contingencias». Explica el uso de toda clase de instrumentos, incluso los lingüísticos, que permite desarrollar sentidos más finos de características de los objetos; desligándolas en tiempo y espacio. Antes, el lenguaje permite una mediación instrumental. Permite relacionar la conducta de otro sujeto con una consecuencia deseada, por ejemplo, al ordenar a alguien que nos traiga algo. En un inicio cuando los objetos están presentes, el referido se contacta con un objeto a través del referidor; ello continúa en ausencia del objeto, y aquí la función referenciadora se desliga de la instrumental. Una etapa siguiente es cuando el marco en que se realiza la interacción condiciona si el otro sujeto seguirá la orden (interacción selectora). Las respuestas entonces se someten a estímulos cambiantes.

Este hecho se ve más claro cuando analizamos las situaciones instrumentales no lingüísticas, que presentan también como rasgo el desarrollo de sentidos. En estas situaciones se dan respuestas de «significación» ante los objetos en base a las cuales el sujeto va a continuar actuando. Los sujetos dan a los objetos una significación, un sentido, a partir del cual se hará una conducta posterior.

En la comunicación, el referidor tiene que haber desarrollado sentidos de los objetos para que pueda emitir palabras pertinentes. Lo que haga el referido luego de acuerdo a estas palabras, es decir, las conductas subsiguientes, es de acuerdo a su entendimiento. Los actos de significación también pueden ayudar a comprender el papel regulador del lenguaje sobre nuestra propia conducta, pero aquí las palabras no constituyen estímulos para las conductas subsiguientes; son las conductas de significación las que intervienen, asumiendo la forma de palabras para dar una significación a los objetos.

\section{La Definición del Pensamiento}

El pensamiento implica lo siguiente:

1. Se dan transformaciones de contingencias propias no lingüísticas y que son sustitutivas en términos de transformar las contingencias presentes en base a otras no presentes.

2. La transformación de contingencias implica el desarrollo de nuevos sentidos ante determinadas situaciones, ya sean problemas u otras. Ello explica el uso de toda clase de instrumentos, incluso los Lingüísticos. Por ejemplo, en una situación problema se desarrollan nuevos sentidos a fin de que ante tal situación se pueda reaccionar de forma efectiva. Asimismo, permite entender como se puede «pensar en grupo», puesto que lo importante es desarrollar nuevos sentidos.

3. El caso del pensamiento como autosustitución no solo implica un. cambio de sustitución para los otros a una para sí mismo. En la sustitución referencial las contingencias se reparten entre dos sujetos, uno el que media (referidor) y otro que es mediado (referido). Tal repartición desaparece en la autosustitución. La situación como 
resultado cambia pero solo en el punto de que el sujeto ya no relaciona sus respuestas con las de otro sujeto sino solo sus propias respuestas. Pero lo más importante es que un punto continúa: el desarrollo de sentidos. La autosustitución entendida por Ribes solo es el último nivel del pensamiento habiendo ya una constante transformación de las contingencias en las que participa el sujeto. Esto es claro cuando se "piensa" entre dos. Todos, al parecer están en una situación de conversación, discusión, pero, además, están cumpliendo la característica de la autosustitución o pensamiento: cada uno media sus propias respuestas referenciales a través de alguna respuesta referencial que los otros dan. Si bien uno refiere al otro sobre alguna cosa, lo principal que se quiere lograr es algo similar a lo que uno hace cuando «reflexiona». Se desarrollan ideas, sentidos de las cosas.

4. En el pensamiento se dan coordinaciones entre conductas en base a los «indicios» dejados por los objetos y que «indican» como relacionar respuestas para ajustarse a ellos. Los indicios señalan en que plano se debe considerar el objeto con el que se está interactuando. Se puede atribuir a los indicios características más complejas, como simbólicas, lo que permite su trascendencia en tiempo y espacio.

5. Las varias conductas que se dan en el pensamiento se presentan como sucesiones. Estos términos son apropiados pues muchas veces el modo de combinarse las respuestas es poco dirigido y más bien automático. Dado que lo importante es el desarrollo de sentidos, la forma como se establecen las relaciones pasa a segundo plano. Aunque también los sentidos pueden «producirse» como cuando buscamos pistas o escogemos caminos para resolver un problema, pero aún en este caso lo esencial es que los sentidos juntos tengan para el sujeto cierta significación, es- decir, lo esencial es en qué orientación con respecto al problema queda el sujeto luego. Se pueden relacionar sentidos de modo arbitrario pero este evento estaría incompleto si es que no se pueda hacer algo a partir de él. Las relaciones entre conductas en el pensar no implican encadenamiento de conductas. Ribes entiende que las relaciones se dan como «mediaciones» entre conductas sustitutivas. Aunque el concepto de mediación supone un avance al considerar el establecimiento de relaciones entre conductas como una «estructuración» de conductas, sigue implicando relaciones entre conductas. Creemos que el sujeto no relaciona conductas, éstas se suceden unas de otras cuando ante determinado complejo estimulativo, la conducta de significación establece la situación para que ciertas conductas con sentido (pertinentes a la situación). Usando una metáfora, esta conducta de significación inicial es como una luz que ilumina un problema revelando sus características en base a las cuales el sujeto desplegará las conductas con sentido pertinentes. Así, como una luz ilumina un cuarto oscuro, luego de lo cual nosotros podemos movemos dentro, la conducta de significación establece la situación para que se sucedan ciertas conductas. Este primer contacto solo establece la situación, las conductas siguientes operaran cambios en los objetos que tiene como resultado principal el cambio en la postura del sujeto ante tales objetos. Ello establecerá nuevas condiciones ante las cuales se seguirán otras conductas.

6. Finalmente proponemos una idea tentativa: en el pensar se dan conductas con sentido. Este término permite explicar los casos donde no hay estímulo al que se responde o la respuesta es correspondiente a propiedades de los objetos en absoluto presentes; por tal 
razón una caracterización más adecuada es considerar que dichas conductas, en realidad, tienen su propio sentido. Cuando observamos un objeto y actuamos con respecto a él se producen conductas con sentido hacia tal objeto, por ejemplo, si este objeto es una hoja en blanco tirada en el suelo la conducta con sentido seria recogerla, cualidad que no se puede deducir de su apariencia. Con este término, se asume que el referente ya no es parte de la situación actual, pertenece a la historia interactiva del sujeto, el referente «viene», entonces con la conducta. El referente formó parte de la contingencia que dio sentido a la conducta pero en posteriores situaciones puede ya no estar presente. Podemos entender, así, las situaciones en las cuales se actúa sin haber objetos presentes, ya no recurriendo a estímulos sustitutos. Estos ya no son necesarios para poner un determinado sentido en escena, porque es la propia conducta la que tiene el sentido. Cuando se reacciona ante un estímulo sustitutivo se da una respuesta referencial de todo el objeto, es decir, le da al estimulo un sentido. y esto sucede porque los numerosos contactos con los objetos ocasionan cambios en la conducta, que predisponen al sujeto a realizar estos actos de significación. Mas adelante, el sujeto puede generar abiertamente estímulos sustitutivos, como cuando habla en voz alta para ayudarse en una acción, pero las palabras no son estímulos sino actos que indican que significa cada estímulo en la situación emprendida y que hacer con respecto a ellos. En resumen proponemos la siguiente definición:

El pensar es el despliegue de varias conductas implícitas y manifiestas que ponen al sujeto en cierta orientación con respecto a un complejo estimulativo a partir de la cual el sujeto podrá hacer ciertas actividades. Cada orientación es dar un sentido a los objetos, por lo que la presencia de estos no es en todos los casos necesario, su lugar puede ser ocupado por respuesta orientativa lingüística.

\section{Una propuesta metodológica en la investigación del pensamiento}

\section{Fundamentos y Características}

A partir de las ideas propuestas hemos diseñado lo que seria una metodología de investigación del pensamiento que tiene como objetivo la observación o registro de las conductas que intervienen en dicha interacción, así como de las relaciones que se dan entre estas. Si partimos de la idea de que en un evento de pensamiento se suceden varias conductas, un objetivo metodológico seria registrar tales conductas. Cada una de estas conductas pone al sujeto en una relación especial con la situación que enfrenta. Siguiendo la definición que propusimos, el sujeto desarrolla un nuevo sentido de la situación por lo cual el objetivo es registrar como se van dando estos sentidos.

La situación en la que se estudiará dicha interacción será una de solución de problemas. Pero esta tarea se registrará como un proceso en construcción, es decir, se tiene como objetivo registrar como se va elaborando la respuesta. Siguiendo el modelo metodológico recurrido por los enfoques conductistas en la investigación, la tarea tendrá la forma de una igualación a la muestra, en la cual el sujeto construirá una figura igual a la de muestra. Pero las figuras que se presentan como alternativas no son figuras con diferentes grados de similitud con el estimulo de muestra, sino iconos que representan las tareas a ejecutar para que una figura simple se convierta en una igual a la de muestra. Cada icono refleja una conducta que pone en relación distintiva al sujeto ante la situación. 
En las tareas de igualación de la muestra la tarea está diseñada para que de acuerdo a la alternativa seleccionada se infiera que conducta ha tenido lugar: igualación de todas las características, de solo algunas, o diferenciación de todas, además de si siguió criterios de igualación. En el modelo aquí presentado se pueden registrar las conductas en juego pues estas son justamente las alternativas. Cada icono se corresponde con una acción necesaria a realizar para resolver la tarea, aunque no el modo en que esta debe hacerse. Al observar la ejecución, el experimentador sabrá que conductas se seleccionan, cuales se descartan, y de acuerdo a cada ítem, si es capaz de ejecutar conductas complejas que reemplacen a otras mas simples. En cada ejecución, los sujetos no podrán visualizar su construcción por lo que esta queda implícita pero registrable en la selección de iconos.

Se realizó un estudio exploratorio en 9 sujetos. Este estudio compara esta metodología, que llamaremos ARMAR con la de igualación de la muestra de segundo orden, que llamaremos IGUALAR. Se construyeron las pruebas de 20 ítems, en las que el sujeto debería construir una figura que satisfaga los criterios de igualación a una muestra de acuerdo a Identidad (todos los rasgos son iguales a los de la muestra), Semejanza (solo algunos rasgos son iguales a los de la muestra) y Diferencia (todos los rasgos son distintos a los de la muestra). Los diseños de los ítems son los mismos en ambas pruebas pero con colores distintos.

\section{PROCEDIMIENTO}

\section{Fase 1:}

Se presenta la prueba ARMAR 1 en la que los sujetos deberán marcar los iconos con marcadores de pizarra acrílica sobre la pantalla, con un aspa en un tiempo de 20 segundos. En cada ejecución, los sujetos no podrán visualizar su construcción por lo que esta queda implícita pero registrable en la selección de íconos. Tampoco se les dará información de sus aciertos. A la semana siguiente, se presenta la prueba IGUALAR 1 con ítems idénticos a la primera prueba pero sin íconos solo figuras de comparación. Se registran las respuestas y tampoco se les informa de sus resultados.

\section{Fase 2 :}

En una segunda etapa, se añaden ítems auxiliares en una ubicación anterior al ítem donde fallo, esta seria una segunda prueba (ARMAR 2). En la segunda presentación se cambian los colores de todos los ítems para reducir los efectos de aprendizaje. A los días siguientes se presentan los ítems anteriores más los correctivos en la prueba de igualación a la muestra de segundo orden (IGUALAR 2). 


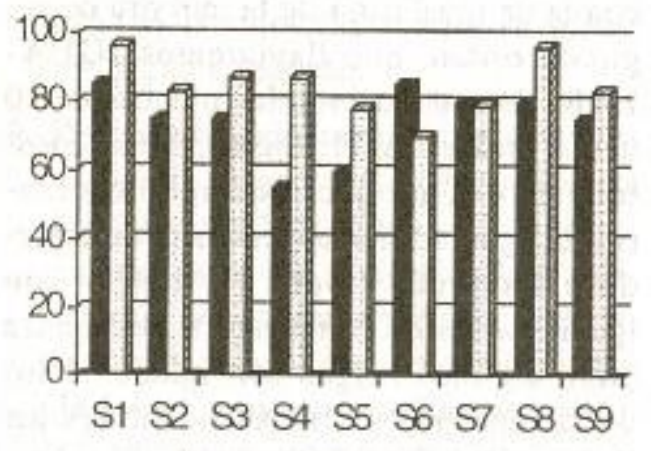

- Armar 1 a Armar 2

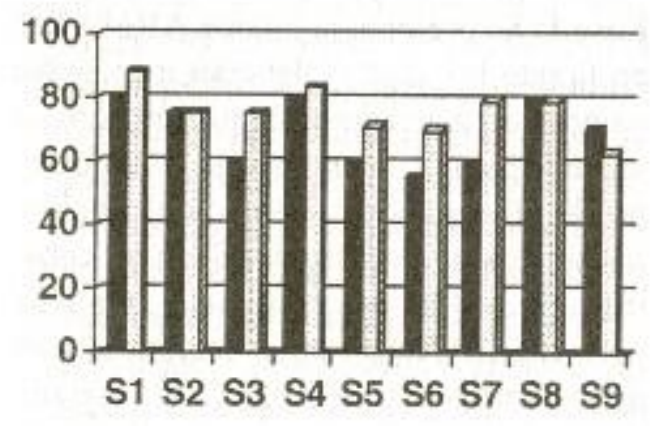

igualacion1 ㅂ.gualacion2

Diferencias estadísticas entre el diseño Armar 1 y 2

\begin{tabular}{|c|c|c|c|c|}
\hline & Armar 1 & Armar2 & Rc & Decís. \\
\hline Prom. & $84,5 \%$ & $74,4 \%$ & 0,82 & Ho \\
\hline
\end{tabular}

Diferencias estadísticas entre el diseño Igualar 1 y 2

\begin{tabular}{|c|c|c|c|c|}
\hline & Igualar 1 & Igualar 2 & Rc & Decís. \\
\hline Prom. & $75,5 \%$ & $68,8 \%$ & 0,49 & Ho \\
\hline
\end{tabular}

Diferencias estadísticas entre el diseño Armar 1 e Igualar 1

\begin{tabular}{|c|c|c|r|c|}
\hline & Armar I & Igualar 1 & Rc & Decis. \\
\hline Prom & $74,4 \%$ & $68,8 \%$ & 0,39 & Ho \\
\hline
\end{tabular}

Diferencias estadísticas entre el diseño Armar 2 e Igualar 2

\begin{tabular}{|c|c|c|c|c|}
\hline Ss & Armar 2 & Igualar 2 & Rc & Decis. \\
\hline Prom & $74,4 \%$ & $68,8 \%$ & 0,77 & Ho \\
\hline
\end{tabular}




\section{Diferencias estadísticas en Mejoras e Involuciones del diseño Armar}

\begin{tabular}{|c|c|c|c|}
\hline & Media & Desviac & Varianza \\
\hline & & & \\
Mejoras & 3,4444444 & 1,58989867 & 2,52777778 \\
Involuciones & 0,7777777 & 1,09290642 & 1,19444444 \\
$T$ & 3,91 & & \\
Sd & 7,96 & & \\
Decisión & Ho & & \\
& & & \\
& & & \\
\hline
\end{tabular}

Diferencias estadísticas en Mejoras e Involuciones del diseño Igualar

\begin{tabular}{|c|c|c|c|}
\hline & Media & Desviac & Varianza \\
\hline & & & \\
Mejoras & 2,5555555 & 1,23603308 & 1,52777778 \\
Involuciones & 1,3333333 & 1 & 1 \\
$T$ & 2,35 & & \\
Sd & 7,96 & & \\
Decisión & Ho & & \\
& & & \\
& & & \\
\hline
\end{tabular}

\section{ANÁLISIS}

Los resultados muestran que en el diseño propuesto (Armar) los sujetos muestran por lo general mayor eficacia: en la fase 1 los porcentajes de acierto se acercan a $80 \%$ en cinco sujetos y $60 \%$ en dos de ellos. Dos llegan a más de $80 \%$. En la fase 2, pasan el $80 \%$ los sujetos 1, 2, 3, 4 Y 8 llegando dos hasta el 96\%. Una eficacia menor se ve en el otro diseño (Igualar). Solo 3 sujetos llegan al $80 \%$ de eficacia en la primera fase. En la fase 2, cinco sujetos elevan su rendimiento levemente y dos pasan el $80 \%$ de eficacia. Los correctivos cumplen una mejor función en el diseño 1 (Armar) pues todos los sujetos tienden a responder correctamente los ítems que en la primera fase respondieron en forma errónea. Cuatro de ellos muestran mejoras importantes 2, 4,5 y 9. Cuatro muestran involución, es decir responden erróneamente un ítem respondido correctamente en la fase 1, aunque solo uno en forma significativa - sujeto 6. En el otro diseño (Igualar), siete sujetos mostraron leves mejoras pero seis mostraron involuciones.

Aplicándose comparaciones estadísticas no se encontró diferencias significativas entre los diseños Armar e Igualar y tampoco entre las fases de cada diseño.

Creemos que el primer diseño permite a los sujetos responder con mayor eficacia pues los iconos ayudan a construir el estimulo que se iguala al estimulo de muestra según el criterio que establecen los estímulos funcionales. El sujeto ya no tiene que elegir entre estímulos de comparación ya acabados el que se iguale a la muestra sino que lo puede ir armándolo con una ayuda visual que son los iconos. Estos últimos serian mediadores, como lo son las conductas que conforman el pensamiento y que permiten al sujeto colocarse en diferentes orientaciones con respecto a un complejo de estímulos al que debe adecuarse. La orientación en una tarea de igualación clásica implica encontrar una figura que se ajuste a criterios de igualdad, diferencia total o similitud parcial, con respecto a la muestra. La 
orientación en el diseño propuesto es ante los estímulos que componen los «estímulos de comparación", es decir, la forma, color, orientación, que en una composición especifica que debe hacer el sujeto, se ajustan a los criterios de Identidad, Diferencia y Similitud. En un caso para encontrar una figura así se tiene que elegir entre alternativas (igualación a la muestra), en el otro caso construir una.

\section{BIBLIOGRAFIA}

Berrocal, P. F y Carretero, M (1995). Perspectivas actuales en el estudio del razonamiento. En M. Carretero, J. Almaraz, P. Fernández: Razonamiento y Comprensión. Madrid: Trota.

Bruner, J. (ed. 1980). Sobre el desarrollo cognitivo. En: Investigaciones sobre el Desarrollo Cognitivo. Madrid, Pablo del Rio.

Carretero, M (1985). Aprendizaje y Desarrollo Cognitivo : un ejemplo del tratado del inútil combate. En M. Carretero y J. Mayor (eds): Actividad Humana y Procesos cognitivos. Madrid, Alhambra.

González, R (1991). Temas de Psicología Cognitiva, Cedeis, Lima Perú.

Kantor, J. R ( 1924-1926) Principles of Psychology. New York: Knopf. Vol. I y II.

Piaget, J. (Ed. 1974). Seis estudios de Psicología. Barcelona: Edit. Six Barral .

Piaget, J. (Ed. 1973). Psicología de la inteligencia .Buenos Aires: Edit. Psique.

Piaget, J. ( 1972 a). El lenguaje y el Pensamiento en el niño. Buenos Aires, Edil. Guadalupe.

Piaget, J. ( 1972 b). El Juicio y el Razonamiento en el niño. Buenos Aires, Edit. Guadalupe.

Ribes, E y López, F (1985). Teoría de la conducta. México, Ed. Trillas.

Ribes, E ( 1990c). Algunos Pensamientos sobre el Pensar y su Motivación. En Psicología General, México, Trillas.

Ribes, E (1990d). Aptitudes Sustitutivas y Planeación del Comportamiento inteligente en instituciones educativas. En Psicología General, México, Trillas

Riviere, A( 1985). Sobre la multiplicidad de las representaciones. Un viaje por los vericuetos de los lenguajes del pensamiento. En J. Mayor (ed), Actividad Humana y Procesos cognitivos. Madrid, Alhambra.

Roca, J ( 1993). Psicología: un enfoque naturalista. Guadalajara, Universidad de Guadalajara.

Ryle, G ( 1967). El Concepto de lo Mental. Ed. Paidos, Bs. As. Argentina.

Skinner, B.F (Ed. 1974). Sobre el conductismo. Barcelona: Fontanella.

Vigotsky, L. ( 1979a ). Pensamiento y lenguaje. Buenos Aires, Edit. Pleyade.

Vigostsky, L. ( I 979b). Las Funciones Psíquicas Superiores. Barcelona, Edit Critica, Grijalbo.

Yela, M. (1985). La Inteligencia y la accion verbal. En M. Carretero y J. Mayor (eds):

Actividad Humana y Procesos cognitivos. Madrid, Alhambra. 